\title{
Bilgisayarlı Tomografide Geriatrik Hastalarda Adrenal İnsidentaloma Prevalansı
}

\author{
The Prevalence of Adrenal Incidentaloma in Geriatric Population \\ Undergoing Computed Tomography
}

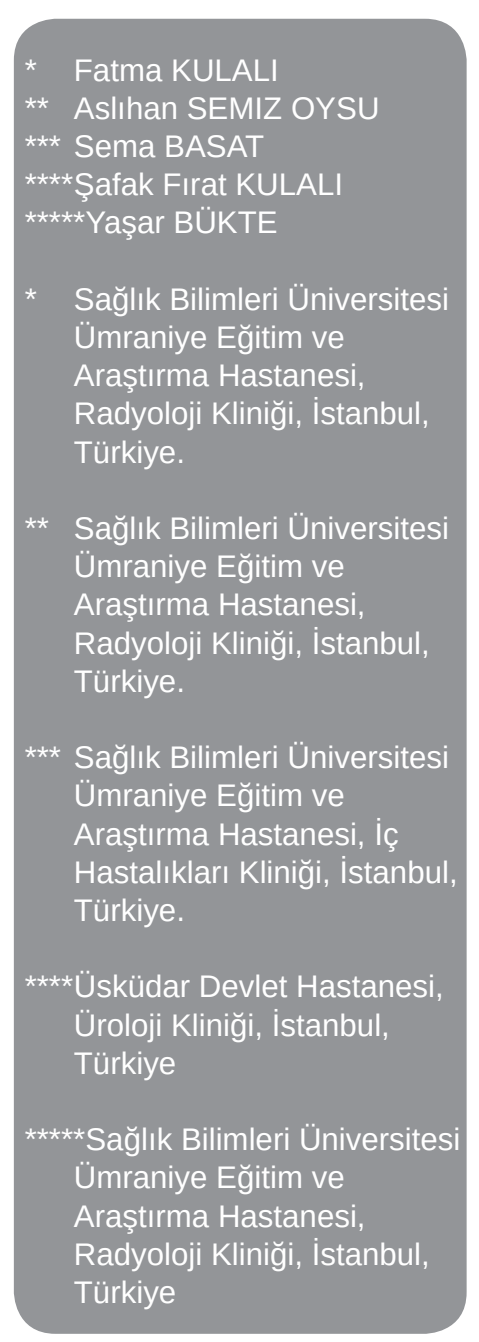

Yazışma Adresi:

Uz. Dr. Fatma Kulalı

Adem Yavuz Cad. No:1, Sağlık

Bilimleri Üniversitesi Ümraniye

Eğitim ve Araștırma Hastanesi,

Radyoloji Kliniği, Ümraniye, 34764,

İstanbul, TÜRKIYE

Telefon: +90 5077391916

ftkulali@gmail.com

\section{Öz}

Amaç: Amacımız, bilgisayarlı tomografi (BT) tetkiklerinde, 65 yaş ve üstü hastalarda insidental saptanan adrenal lezyonların prevalansını araştırmaktır. Gereç ve Yöntem: Hastanemizde, Ocak 2013 ve Nisan 2014 tarihleri arasında, üst batın BT tetkikleri yapılmış ardışık 903 hasta tekrar değerlendirildi. Bilinen kanser öyküsü olan, adrenal patolojilere yönelik tetkik yapılan ve 65 yaş altı hastalar çalışma dışı bırakıldı. Çalışma grubunu, 178 hasta oluşturdu. Çalışma grubunda yer alan hastaların, BT tetkikleri incelendi. Adrenal lezyon varlığı, varsa lezyon en uzun çapı ve görüntüleme özellikleri bir radyolog tarafından kaydedildi. Lezyonların radyolojik bulgularına göre ön tanıları yapıldı. Malignite özellikleri olanlar, çalışma dışı bırakıldı. BT' de, adrenal insidentaloma saptanma oranı hesaplandı. Bulgular: BT tetkikleri incelenen, 93'ü kadın, 85'i erkek olan, 65 yaş ve üzeri 178 hasta çalışma grubunu oluşturdu. Bu hastaların, 86'sında adrenal lezyon saptanırken 92'sinde adrenal glandlar doğaldı. Olguların ikisinde malignite ile uyumlu görünüm saptandığı için insidentaloma grubundan çıkarıldı. İnsidental adrenal lezyon saptanan 84 olgunun, 20'sinde diffüz adrenal hiperplazi, 53'ünde adenom, 11'inde myelolipom ile uyumlu radyolojik özellikler izlendi. Adrenal insidentaloma prevalansı \%47,2 olarak saptandı. Sonuç: Çalışmamızda, BT'de geriatrik hastalarda adrenal insidentaloma prevalansı \%47,2 bulunmuş olup küçümsenmeyecek derecededir. Günümüzde görüntüleme yöntemlerinin daha çok kullanılması ile sık saptanan adrenal insidentalomaların malignite ekartasyonu yapıldıktan sonra fonksiyonel açıdan değerlendirilmesi gereklidir.

Anahtar Kelimeler: Adrenal, bilgisayarlı tomografi, geriatrik, insidentaloma

\begin{abstract}
Object: The aim of our study was to estimate the prevalence of adrenal incidentaloma in geriatric patients who underwent abdominal computed tomography (CT). Methods: In a single institution, between January 2013 and April 2014, consecutive 903 patients undergoing abdominal computed tomography were reviewed. Among them, patients with previous malignancy, patients with suspicion of adrenal gland disease and $<65$ years-old patients were excluded. The remaining 178 patients were enrolled in this study. Abdominal CTs of 178 patients were evaluated from PACS (Picture Archiving and Communications System). The existence of adrenal lesion, the longest diameter and imaging findings of lesion were noted by a radiologist. The prevalence of
\end{abstract}


adrenal incidentaloma was estimated. Results: The images of 178 (95 women, 85 men) patients were evaluated for adrenal gland lesions. All of them were $\geq 65$ years-old. Of 178, 86 had adrenal lesions and 92 had normal adrenal glands. There were 2 patients with malignant adrenal lesions. They were also excluded from incidentaloma group. In incidentaloma group $(n=84), 20$ had diffuse adrenal hyperplasia, 53 had adrenal adenoma and 11 had myelolipoma according to CT findings. The prevalence of adrenal incidentaloma was found as 47.2\%. Conclusion: Nowadays, imaging modalities are widely performed. So, diagnosis of adrenal incidentaloma is increasing. For adrenal incidentaloma, a high prevalence of $47.2 \%$ was observed among geriatric population who underwent CT in our study.

Keywords: Adrenal, computed tomography, geriatric, incidentaloma.

\section{Giriş}

Adrenal insidentalomalar, adrenal dışı sebeplerle yapılan görüntüleme yöntemleri ile tesadüfen saptanan lezyonlardır. Günümüzde, ultrasonografi (USG), bilgisayarlı tomografi (BT) ve manyetik rezonans görüntüleme (MRG) gibi radyolojik tetkiklerinin kullanımının yaygınlaşması sonucu, adrenal insidentalomalarla daha sık karşılaşılmaktadır (1-3). BT, adrenal gland değerlendirmesinde etkili bir tetkiktir. Adrenal gland boyutlarının küçük olması, BT' de ince kesitlerin alınabilmesi, multiplanar görüntülemeye olanak tanıması ve maliyetinin MRG' ye göre düşük olması nedeniyle BT, adrenal gland lezyonlarının değerlendirilmesinde tercih edilen önemli bir tetkiktir (2-4).

Adrenal insidentalomalar, 65 yaş ve üzeri olgularda daha yüksek oranda saptanmaktadır (5-7). Bizim amacımız, BT' de geriatrik hastalarda adrenal insidentaloma saptama prevalansını araştırmaktır.

\section{Yöntem}

Hastanemizde, Ocak 2013 ve Nisan 2014 tarihleri arasında, üst batın BT tetkiki yapılmış ardışık 903 hasta, hastane bilgi yönetim sistemi (HBYS) kullanılarak incelendi. Çalışma öncesinde etik kurulunun yazılı onayı alındı ve Helsinki Deklarasyonu Kriterleri'ne uygun olarak hastaların kimlik ve sağlık bilgileri korundu. Altmış beş yaş altı olan hastalar $(n=594)$, çalışma dışı bırakıldı. Kalan 65 yaş ve üzeri hastalardan adrenal gland patolojilerine yönelik BT tetkiki olan $(n=21)$ ve bilinen kanser öyküsü olan hastalar $(n=115)$ da çaIışmaya alınmadı. Altmış beş yaş ve üzeri olan, 178 hasta çalışma grubunu oluşturdu. Çalışma grubunda yer alan hastaların BT tetkikleri, görüntü arşivleme ve iletişim sistemleri (PACS - Picture Archiving Communication Systems) kullanılarak tekrar gözden geçirildi. Tüm BT tetkikleri, çok kesitli helikal BT (Somatom Sensation 40, Siemens Sağlık Sektörü, Erlangen, Almanya) ile yapılmıştı. Kontrastsız ve intravenöz (i.v.) kontrastlı BT için tarama parametreleri şu şekildedir: tüp voltajı 120 kVp; tüp akımı, 20 mAs, helikal kalınlık, 2,5 mm, interval, 2,5 mm, pitch, 1,5 ve rekonstrüksiyon kesit kalınlığı 1,25 mm.

Tüm hastaların kontrastsız BT tetkiki veya portal venöz fazda (i.v. kontrast madde sonrası 70-90 saniyede) yapılmış i.v. kontrastlı BT tetkiki mevcuttu. Adrenal lezyon varlığı, en uzun çapı ve görüntüleme özellikleri (sağ/sol/bilateral yerleşim, dansitesi, düzgün/düzensiz kontur, homojen/heterojen olması) bir radyolog tarafından kaydedildi. Lezyonun dansitesi, iş istasyonunda (Leonardo, Siemens Sağlık Sektörü) ilgilenilen bölge (ROI- region of interest) ölçümleri yapılarak Hounsfield ünitesi $(\mathrm{HU})$ olarak hesaplandı. Her lezyon için, lezyonun boyutuna göre 8 - 20 mm2 arasında değişen büyüklükte ayrı lokalizasyonlardan 3 ayrı ROI ölçümü yapıldı ve ortalamaları lezyonun dansitesi olarak kabul edildi. Lezyonların radyolojik bulgulara göre ön tanıları yapıldı. Kontrastsız BT tetkikinde, 3 cm' den küçük ve dansitesi <10 HU olan veya portal venöz fazda alınan kontrastlı BT' lerde dansitesi < 37 HU olan homojen ve düzgün konturlu lezyonlar radyolojik olarak adenom olarak kaydedildi $(1,6,8)$. En az 6 aylık takiplerinde, radyolojik olarak anlamlı değişiklik göstermeyen yumuşak doku dansitesindeki, düzgün konturlu ve homojen nodüler lezyonlar da adenom olarak kabul edildi. Makroskopik yağ içeriği ( $\leq-10 \mathrm{HU}$ ) olan, büyük heterojen lezyonlar, radyolojik olarak myelolipom olarak raporlandı (Şekil 1). Adrenal gland korpusunun ve kruslarının kalınlığı simetrik diffüz olarak $\geq 10 \mathrm{~mm}$ ise radyolojik olarak adrenal hiperplazi olarak not edildi $(1,8)$. BT'de dansitesi yüksek, heterojen ve düzensiz konturlu lezyonlar, malignite açısından anlamlı kabul edildi. Malignite düşünülen hastaların, daha sonraki tetkikleri primer hastalık veya progresyon açısından incelendi. Primer hastalık tespit edilen veya progresyon gösteren hastalarda malignite tanısı doğrulandı. Malignite görünümü olanlar çalışma dışı bırakıldı. Çalışma grubu, 
radyolojik olarak normal, adrenal hiperplazi, adenom ve myelolipom olarak 4 grupta incelendi. Lezyonların, takip BT tetkikleri ile karşılaştırılması yapılarak değişiklik olup olmadığı kaydedildi. En son çekilen takip BT ile çalışma için değerlendirilen BT arasında geçen zaman not alındı. Ortalama takip süreleri hesaplandı. Çalışma grubunda, adrenal lezyon saptanma oranı prevalans hesaplama formülü ile hesaplandı. Parametrelerin normal dağılıma uygunluğu Shapiro Wilks testi ile değerlendirilmiştir. Niceliksel verilerin karşılaştırılmasında normal dağılım gösteren parametrelerin iki grup arası karşılaştırmalarında Student t testi ve niteliksel verilerin karşılaştırılmasında ise Ki Kare testi kullanıldı. Anlamlılık p<0,05 düzeyinde değerlendirildi.

\section{Bulgular}

Çalışmada, BT tetkikleri incelenen 65 yaş ve üzeri 178 hasta [93(\%52,2) kadın, 85(\%47,8) erkek] yer al-

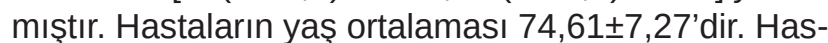
taların 86'sında adrenal lezyon saptanırken, 92'sinde adrenal glandlar doğaldı. Çalışma grubunda, adrenal lezyonu olanlar ve olmayanlar arasında yaş ortalamaları ve cinsiyet dağılımı açısından istatistiksel olarak anlamlı bir farklılık saptanmadı $(p>0,05)$.

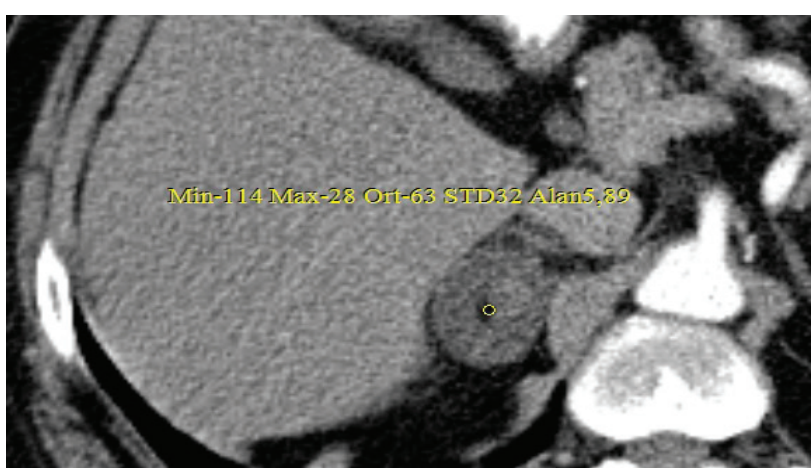

Şekil 1: 76 yaşında kadın hastada intravenöz kontrastlı BT tetkikinde sağ adrenal glandda 3,5x3 $\mathrm{cm}$ boyutlarında myelolipom görünümü.

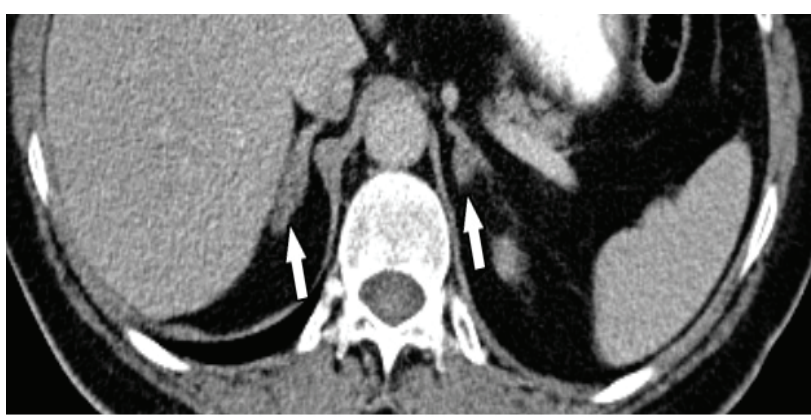

Hastaların ikisinde, metastaz ile uyumlu görünüm saptandığı için insidentaloma grubundan çıkarıldı. Metastazı olduğu düşünülen hastaların sonraki tetkiklerinde, bir hastada kolon kanseri ve diğer hastada akciğer kanseri tespit edildi. Insidental adrenal lezyon saptanan 84 (\%47) hastanın, 20'sinde adrenal hiperplazi, 11'inde myelolipom, 53' ünde adenom ile uyumlu radyolojik özellikler izlendi (Şekil 2). Çalışma grubunda adrenal insidentaloma prevalansı yaklaşık \%47,2 [güven aralığı (GA) \%95; 39,68-54,80] olarak hesaplandı. Adrenal lezyonlar, hastaların \%61'inde sol tarafında, \%29'unda sağ tarafında ve \%10'unda bilateral yerleşimliydi (Şekil 3). Adrenal insidentalomaların çoğu sol taraf yerleşimliydi. Adenom, myelolipom ve adrenal hiperplazi grupları arasında sağ/sol taraf yerleşimi açısından istatistiksel olarak anlamlı farklılık saptanmadı ( $p>0,05)$. Adrenal lezyon saptanan geriatrik grupta, ortalama en uzun çap, BT' de ön tanısı adenom olanlar için, $14 \pm 5,37 \mathrm{~mm}$ (10-30mm) ve myelolipom olanlar için, $20 \pm 6,69 \mathrm{~mm}$ (14 -35mm) olarak hesaplandı. Myelolipom grubunda ortalama dansite $-22,09 \pm 10,75 \mathrm{HU}$ (-10 ile $-40 \mathrm{HU}$ arasında) saptanırken adrenal adenom grubunda ortalama dansite $8,19 \pm 7,39 \mathrm{HU}$ (-11 ile $30 \mathrm{HU}$ arasında) olarak bulundu (Şekil 4).

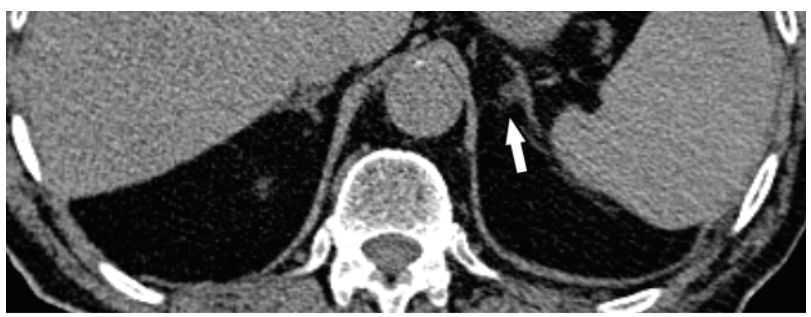

Şekil 2: 66 yaşında kadın hastada kontrastsız BT tetkikinde sol adrenal glandda ortalama $5 \mathrm{HU}$ dansitesinde adenom ile uyumlu görünüm (beyaz ok).

Şekil 3: 65 yaşında kadın hastada intravenöz kontrastlı BT tetkikinde bilateral adrenal adenom görünümü (beyaz oklar). 


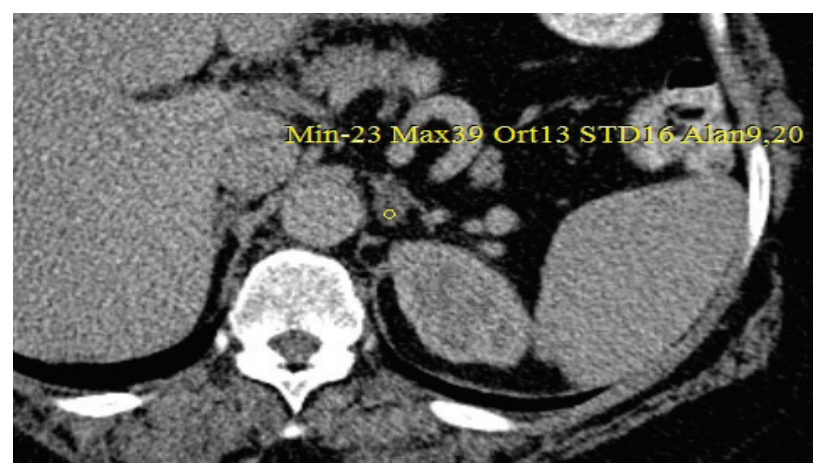

İncelenen BT tetkiki ile en son çekilen takip BT tetkiki

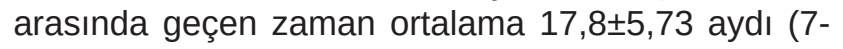
28 ay).Takip BT' lerinde, adrenal insidentalomaların hiçbirinde anlamlı değişiklik izlenmedi.

\section{Tartışma}

Günümüzde, insidental saptanan adrenal kitle sıklığı artmıştır (1-3). Saptanan insidental adrenal lezyonların, öncelikle benign- malign ayrıcı tanısı yapılmalı ve fonksiyonel olup olmadıkları araştırılmalıdır. Adrenal insidentalomaların çoğu, nonfonksiyoneldir (7). Fonksiyonel unilateral insidentaloma varlığında, sıklıkla ilk tedavi seçeneği adrenalektomidir (6, 9-13). Nonfonksiyonel insidentalomalarda, benign-malign ayırımı için lezyonun boyutu, görüntüleme özellikleri ve büyüme hızı değerlendirilmelidir. BT veya MRG' nin adrenal lezyonların saptanmasında ve değerlendirilmesinde önemli bir rolü vardır (2-4). İnsidentalomaların çapı 4 cm' den küçük olanların \%60'ından fazlası benign adenomdur, primer adrenal karsinom \%2' den azdır. Ancak, $6 \mathrm{~cm}$ 'den büyük lezyonlarda malignite riski \%25'e yükselir (6). Bu sebeple, 6 cm'den büyük lezyonlara adrenalektomi önerilir (6, 9-13). Görüntüleme bulguları benign özellikler gösteren $4 \mathrm{~cm}$ ' den küçük lezyonlara takip önerilmektedir. Çapları 4-6 cm arasında yer alan lezyonlarda, görüntüleme özelliklerine ve hastanın kliniğine göre, takip veya adrenalektomi kararı verilmelidir. Yapılan küçük serili çalışmalarda, 10 yıllık takiplerde insidentalomaların \%30'dan azında boyut artışı ve \%20'sinden azında biyokimyosal anormallikler tespit edilmiştir (6). Bu nedenle, klinisyenler, lezyonun görüntüleme bulgularına, hastanın bireysel özelliklerine ve kliniğine göre tedavi seçeneğinin belirlenmesini önermektedir (6, 9-13).

Adrenal insidentaloma prevalansı, çalışmalar arasında değişkenlik göstermektedir. Arnold ve arkadaşları,
Şekil 4: 71 yaşında erkek hastada kontrastlı BT tetkikinde sol adrenal glandda adenom görünümü.

batın BT taramalarında saptanan adrenal kitle insidansının yaklaşık \%0,6 -\%1,3 oranında olduğunu belirtmişlerdir (2). Şen ve arkadaşlarının yaptığı bir çaIışmada, hem toraks ve batın BT, hem de batın MRG tetkikleri olan geniş bir popülasyonda, adrenal insidentaloma prevalansı \%5,9 bulunmuştur. Ancak, geriatrik hasta popülasyonu ayrıca değerlendirilmemiştir. Tabarin tarafından yapılan bir çalışmada, batın BT tetkiklerinde yaklaşık \%2-3 oranında adrenal insidentaloma saptandığı ifade edilmiştir (14). Kapsamlı bir literatür taraması yapan Barzon ve arkadaşlarının çalışmasında, adrenal insidentaloma prevalansının otopside \%2,3 ve BT' de\% 0,5-2 oranında olduğu bildirilmiştir (15). Davenport ve arkadaşlarının yaptığı bir başka çalışmada, adrenal insidentaloma prevalansı \%0,81-0,98 arasında bulunmuştur (16).

Geriatrik popülasyonda, insidentaloma sıklığının arttığı bilinmektedir (5-7). Grumbach ve arkadaşları, otopside saptanan adrenal insidentaloma prevalansının 30 yaş ve altı hastalarda \%1'den az olduğunu ve 70 yaş ve üzeri hastalarda \%7'ye yükseldiğini belirtilmiştir (6). Literatürde, geriatrik popülasyonda adrenal insidentaloma prevalansına yönelik veri oldukça azdır. Bu sebeple, çalışmamızda, ülkemizde geriatrik bir hasta grubunda adrenal insidentaloma prevalansını araştırdık.

Çalışmamızın avantajları, tek merkezli olması, aynı BT cihazı ile çekilmiş belli bir zaman dilimindeki ardışık 65 yaş ve üzeri hasta grubunda yapılmış olmasıdır.

Çalışmamızın limitasyonlarından biri, insidentaloma saptanan hastaların laboratuvar özellikleri incelenerek fonksiyonel/nonfonksiyonel adenom ayırımı için ayrı bir değerlendirme yapılmamış olmasıdır. Diğer bir limitasyonu ise, lezyonların histopatolojik tanıları- 
nın olmaması ve altın standart bir yöntemle doğrulanmamış olmasıdır.

Sonuç olarak, çalışmamızda BT' de geriatrik hastalarda adrenal insidentaloma prevalansı \%47,2 bulunmuş olup küçümsenmeyecek derecededir. Günümüzde görüntüleme yöntemlerinin daha çok kullanılması ile sık saptanan adrenal insidentalomaların, malignite ekartasyonu yapıldıktan sonra fonksiyonel açıdan değerlendirilmesi gereklidir.

Çıkar Çakışması: Yoktur.

Fonlama Kaynakları: Destekleyen fon veya kuruluş yoktur.

\section{Kaynaklar}

1. Bada M, Castellan P, Tamburro FR, Berardinelli F, Neri F, Cindolo L, et al. Work up of incidental adrenal mass: state of the art. Urologia. 2016;18;83(4):179185.

2. Arnold DT, Reed JB, Burt K. Evaluation and management of the incidental adrenal mass. Proc Bayl Univ Med Cent. 2003;16(1):7-12.

3. Neslihan Başcıl Tütüncü. Adrenal insidentalomalar.Turk J Endocrinol Metab 2003;7.

4. Connolly MJ, McInnes MD, El-Khodary M, McGrath TA, Schieda N. Diagnostic accuracy of virtual non-contrast enhanced dual-energy CT for diagnosis of adrenal adenoma: a systematic review and meta-analysis. Eur Radiol. 2017;13.

5. Li J, Ji Z, Huang Z. Clinical diagnosis and treatment of adrenocortical adenoma in patients aged 60 years or above. Zhonghua Wai Ke Za Zhi. 2016;1;54(2):1336.

6. Grumbach MM, Biller BM, Braunstein GD, Campbell KK, Carney JA, Godley PA, et al. Management of the clinically inapparent adrenal mass ("incidentaloma"). Ann Intern Med. 2003;4;138(5):424-9.

7. Sen H, Asik M, Uysal F, Binneloglu E, Gunes F, Erbag G, et al. The prevalence of incidental adrenal mass found using diagnostic imaging techniques. Turk Jem. 2013; 17: 108-10.

8. Wale DJ, Wong KK, Viglianti BL, Rubello D, Gross MD. Contemporary imaging of incidentally discovered adrenal masses. Biomed Pharmacother. 2017; 87: 256-262.

9. Comlekci A, Yener S, Ertilav S, Secil M, Akinci B, Demir T, et al. Adrenal incidentaloma, clinical, metabolic, follow-up aspects: single centre experience. Endocrine. 2010;37(1):40-6.
10.Muth A, Hammarstedt L, Hellström M, Sigurjónsdóttir HÁ, Almqvist E, Wängberg B. Adrenal study group of Western Sweden. cohort study of patients with adrenal lesions discovered incidentally. $\mathrm{Br} \mathrm{J}$ Surg. 2011; 98(10):1383-91.

11. Bin X, Qing Y, Linhui W, Li G, Yinghao S. Adrenal incidentalomas: experience from a retrospective study in a Chinese population. Urol Oncol. 2011;29(3):270-4. 12. Kapoor A, Morris T, Rebello R. Guidelines for the management of the incidentally discovered adrenal mass. Can Urol Assoc J. 2011;5(4):241-7.

13. Al-Thani $\mathrm{H}$, El-Menyar A, Al-Sulaiti M, ElGohary $\mathrm{H}$, Al-Malki A, Asim M, et al. Adrenal mass in patients who underwent abdominal computed tomography examination. N Am J Med Sci. 2015;7(5):212-9.

14. Tabarin A. Adrenal incidentalomas. Presse Med. 2014; 43: 393-400.

15.Barzon L, Sonino N, Fallo F, Palu G, Boscaro M. Prevalence and natural history of adrenal incidentalomas. Eur J Endocrinol. 2003; 149(4):273-85.

16. Davenport C, Liew A, Doherty B, Win HH, Misran $\mathrm{H}$, Hanna S, et al. The prevalence of adrenal incidentaloma in routine clinical practice. Endocrine. 2011; 40(1):80-3. 